\title{
SOFTMAT-OA: OBJETO DE APRENDIZAGEM PARA FORMAÇÃO DE PROFESSORES DE MATEMÁTICA
}

\author{
Gilmara Teixeira Barcelos, PGIE/UFRGS, CEFET Campos/RJ \\ gilmarab@cefetcampos.br \\ Silvia Cristina Freitas Batista, PGIE/UFRGS, CEFET Campos/RJ \\ silviac@cefetcampos.br \\ Patricia Alejandra Behar, NUTED-PGIE/UFRGS \\ patricia.behar@ufrgs.br
}

\section{RESUMO}

As Tecnologias de Informação e Comunicação (TICs) podem contribuir, de forma significativa, para o processo de ensino e aprendizagem. No entanto, utilizar estas tecnologias para fins educacionais implica reflexões e análises, e engendra a necessidade de formação adequada dos professores. Visando a colaborar nesse sentido, foi desenvolvido o objeto de aprendizagem SoftMat-OA, para subsidiar a preparação de professores de Matemática com relação ao uso pedagógico das TICs. Trata-se de um objeto fundamentado na concepção construtivista/interacionista, desenvolvido em HTML, contendo tutoriais, atividades, animações, applets, entre outros recursos, para apoiar uma oficina pedagógica relacionada ao uso de softwares na aprendizagem de Matemática. Este artigo apresenta o SoftMat-OA, seus objetivos, as concepções pedagógicas que embasaram seu desenvolvimento e sua estrutura. São descritas as fases de concepção, planificação e implementação do objeto. Apresentam-se algumas de suas telas e destacam-se critérios de usabilidade considerados no seu desenvolvimento. Finalizando, analisa-se uma primeira avaliação do objeto.

Palavras-chave: SoftMat-OA, Objeto de Aprendizagem, Formação de Professores, Matemática.

\section{SOFTMAT-0A: LEARNING OBJECT FOR MATH TEACHER'S EDUCATION PROGRAMS}

\begin{abstract}
Information and Communication Technologies (ICTs) can give a significant contribution to teaching and learning processes. However, using these technologies for educational purposes requires not only considerations but also the necessity of adequate teachers' education programs. With the purpose of collaborating for this issue, the learning object SoftMat-OA was developed to introduce and support future Math teachers in pedagogical uses of technologies. The object - based on constructivist/interactionist theoretical principles - was developed in HTML, with tutorials, activities, animation, applets, among other resources, aiming at supporting a pedagogical workshop related to the use of software in Math learning. This article describes SoftMat-OA, its planning, objectives, implementation, and the underlying pedagogical concepts used in its design. Some screens and the usability criteria used in its development are also shown. The final section presents the first evaluation of the object.
\end{abstract}

Key Words: SoftMat-OA, Learning Object, Teacher's Education, Mathematics. 


\section{Introdução}

D’Ambrósio (2001) destaca que a educação para a cidadania é um dos grandes objetivos da educação atual, sendo necessário levar em consideração o conhecimento moderno, impregnado de ciência e tecnologia. A Matemática ensinada nas escolas é, segundo esse autor, ultrapassada, antiga e descontextualizada, o que causa desinteresse por parte dos alunos.

O resultado da avaliação realizada em 2006, no Brasil, pelo Programa Internacional de Avaliação de Alunos (PISA) sinaliza a necessidade de reformulações no ensino de Matemática no Brasil. Em 2006, 9.295 estudantes brasileiros, na faixa de 15 anos (do $7^{\circ}$ e $8^{\circ}$ anos do Ensino Fundamental e no $1^{\circ}$ e no $2^{\circ}$ anos do Ensino Médio) participaram da avaliação do PISA. Numa escala de 0 (zero) a 800, os estudantes brasileiros obtiveram uma média de 369,52 pontos na prova de Matemática. Com esse desempenho, o Brasil ficou em $54^{\circ}$ lugar em Matemática, numa prova da qual participaram 57 países (INEP, 2007).

O espaço ocupado pela Matemática nas escolas deve ser bem aproveitado e, nesse sentido, diversas ações podem ser empreendidas. As Tecnologias de Informação e Comunicação (TICs), utilizadas em atividades de investigação, podem ser recursos favoráveis para aprendizagem de Matemática, uma vez que reforçam o papel da linguagem gráfica e de novas formas de representação, e relativizam a importância do cálculo e da manipulação simbólica (Ponte; Oliveira; Varandas, 2003).

Em particular, a Internet, pode ser muito útil em termos educacionais, favorecendo a construção cooperativa, o trabalho conjunto entre professores e alunos, próximos física ou virtualmente (Moran, 2002). Além disso, pode ajudar a desenvolver a intuição, a flexibilidade mental e a adaptação a ritmos diferentes.

A Internet e os softwares educacionais podem colaborar para o processo de ensino e aprendizagem de Matemática. Como defendido por Baldin (2002), a utilização dos recursos tecnológicos na Matemática é motivada por algumas facilidades que estes podem trazer, tais como: capacidade computacional, visualização gráfica, descoberta e confirmação de propriedades, possibilidades de executar experimentos com coleta de dados e modelagem de problemas, especulações, entre outras.

A ação do professor é fundamental para que ocorram melhorias no processo de ensino e aprendizagem. Além disso, segundo Vygotsky (2007), a mediação por meio de instrumentos e signos no contexto social, possibilita o desenvolvimento dos processos psicológicos superiores. Defende-se, então, que as TICs, utilizadas como instrumentos, por professores preparados e dispostos a utilizar as potencialidades destes, podem favorecer o processo de ensino e aprendizagem.

Um fator imprescindível para a utilização das TICs como recurso didático, portanto, é a formação de professores, tanto inicial quanto continuada. Segundo Sette, Aguiar e Sette (1999), as licenciaturas devem formar profissionais que não só possuam domínio das TICs, mas que também tenham condições de incorporá-las ao processo de ensino e aprendizagem.

Barcelos (2004) afirma que o uso do computador na educação pode enriquecer o processo de aprendizagem matemática, favorecendo a construção de conhecimentos. Porém, a autora defende que os professores de Matemática devem estar bem preparados, o que implica uma boa formação inicial, ou seja, é fundamental o desenvolvimento de estratégias de ação que propiciem a inclusão digital de professores de Matemática, incentivando o uso adequado das TICs em práticas pedagógicas. Ressalta-se que "inclusão digital não é só o amplo acesso à tecnologia, mas a apropriação dela na resolução de problemas" (Fagundes, 2004, p.26). 
Nesse contexto, o presente artigo descreve o objeto de aprendizagem SoftMat-OA, apresentando seus objetivos, as concepções pedagógicas que embasaram seu desenvolvimento, e sua estrutura. Para tanto, na seção 2 apresenta-se o SoftMat-OA como um objeto de aprendizagem fundamentado na pedagogia construtivista/interacionista e são descritas as fases de concepção, preparação e implementação do projeto. Na seção 3, descreve-se a primeira avaliação do objeto, realizada por um grupo de doutorandos. Finalizando, a seção 4 apresenta algumas considerações finais sobre o trabalho desenvolvido.

\section{Construindo o objeto de aprendizagem SoftMat-OA: material e métodos}

Ações que possibilitem a inclusão digital de licenciandos e professores de Matemática são fundamentais. Sendo assim, foi desenvolvido o objeto de aprendizagem SoftMat-OA (http://www.es.cefetcampos.br/softmat/Softmatoa), com o propósito de contribuir na formação, dos referidos licenciandos e professores, para uso pedagógico das TICs. O objeto é composto de páginas HTML, contendo tutoriais, atividades, animações, applets, entre outros recursos, e visa a apoiar, como material educacional digital, uma oficina pedagógica direcionada a professores e licenciandos em Matemática. A referida oficina denomina-se "Softwares Educacionais no Processo de Ensino e Aprendizagem de Matemática" e tem os seguintes objetivos:

- estimular o uso das Tecnologias de Informação e Comunicação (TICs), de forma interativa, nas aulas de Matemática;

- identificar, analisar e propor formas de ação que possibilitem relacionar competências e habilidades de Matemática com as TICs;

- apresentar e caracterizar quatro softwares gratuitos para Matemática (em português), assim como, propor formas de utilização para os mesmos.

O nome SoftMat-OA decorre de o fato do mesmo ser um objeto de aprendizagem (OA), destinado a apoiar uma oficina pedagógica envolvendo softwares de Matemática. Compreende-se um objeto de aprendizagem como "qualquer recurso digital que possa ser reutilizado para dar suporte ao ensino" (Wiley, 2001, p. 7). Qualquer material eletrônico que contenha informações que possibilitem a construção do conhecimento pode ser considerado um OA, seja essa informação em forma de imagem, página HTML, animação ou simulação (RIVED, s.d).

Segundo Macêdo et al. (2007), não há um consenso, universalmente aceito, sobre a definição de um OA: podem ser objetos criados em qualquer mídia ou formato e podem ser simples, como uma animação ou uma apresentação de slides; ou complexos, como uma simulação. Podem, de acordo com Bettio e Martins (2004), utilizar imagens, animações, applets, arquivos de texto ou hipertexto, dentre outros. Definições amplas, como as apresentadas, são adequadas para o SoftMat-OA, que é desenvolvido em HTML, nos moldes de uma unidade de aprendizagem.

A principal característica de um OA é a reusabilidade, que possibilita incorporá-lo em várias aplicações. Segundo Tarouco e Dutra (2007), a idéia básica é a de que os OAs sejam como blocos com os quais será construído o contexto de aprendizagem.

O SoftMat-OA é composto de diversas páginas HTML e adaptá-lo para subsidiar oficinas sobre outros temas, ou mesmo utilizar algumas de suas páginas em outros contextos não é difícil. A estrutura do SoftMat-OA já foi adaptada, por exemplo, para apoiar um minicurso sobre applets de Trigonometria.

$\mathrm{Na}$ elaboração do SoftMat-OA procurou-se atender a critérios tecnológicos, a concepções pedagógicas, a práticas metodológicas e a critérios de usabilidade. O SoftMat-OA fundamenta-se na concepção construtivista/interacionista, ou seja, nas perspectivas piagetiana (que enfatiza a interação do sujeito com o objeto) e 
vygostskiana (que enfatiza a interação do sujeito com o outro, num contexto social). Nesta concepção, o conhecimento é construído a partir da ação do sujeito sobre o objeto de conhecimento, interagindo com ele, sendo as trocas sociais condições necessárias para aprendizagem. $\mathrm{O}$ aluno participa ativamente da própria aprendizagem, mediante a experimentação, a pesquisa em grupo, o estímulo à dúvida e o desenvolvimento do raciocínio, entre outros procedimentos.

Para que um experimento seja um meio efetivo para estudar "o curso do desenvolvimento de um processo", este deve oferecer o máximo de oportunidades para que o sujeito experimental se engaje nas mais variadas atividades que possam ser observadas e não apenas rigidamente controladas (Vygotsky, 2007). Nesse sentido, o SoftMat-OA foi estruturado visando a possibilitar diversas atividades, por meio de recursos variados.

O SoftMat-OA foi desenvolvido em três etapas principais: concepção do projeto, planificação e implementação. Na etapa de concepção do projeto foram definidos os seus principais objetivos, o público-alvo (professores e licenciandos em Matemática) e os pressupostos teóricos que o embasariam. Na planificação, definiu-se que o objeto seria desenvolvido em HTML, com animações em Flash. A partir dessas definições, foi elaborado o storyboard do objeto (Figura 1), visando a orientar o seu desenvolvimento. Este permitiu, não só organizar seções e subseções, mas também organizar os conteúdos e recursos a serem disponibilizados nas mesmas.

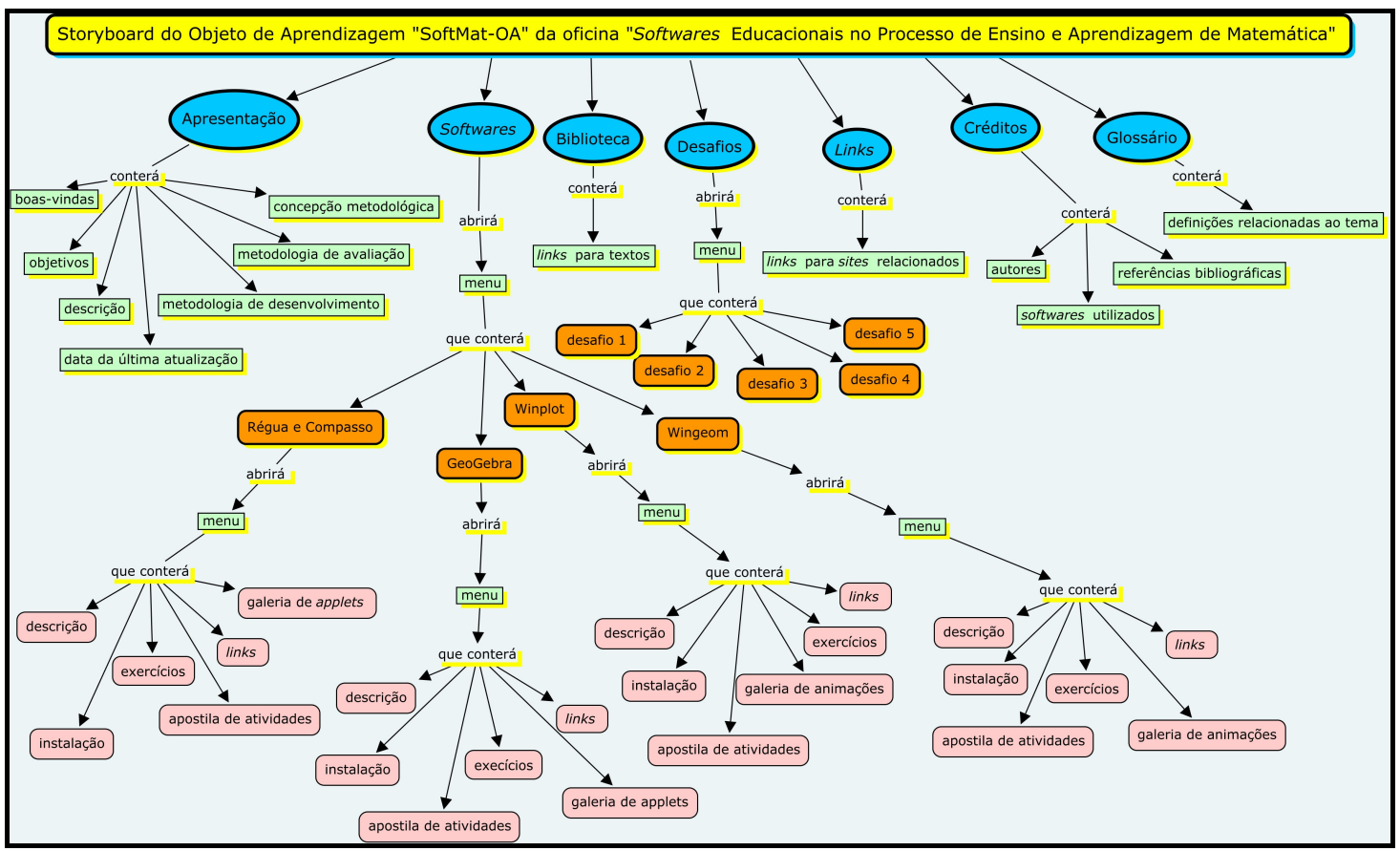

Figura 1 - Storyboad do SoftMat-OA

$\mathrm{Na}$ fase de implementação definiram-se, inicialmente, os softwares a serem utilizados:

- NVU (http://nvudev.com/index.php) - desenvolvimento das páginas HTML;

- Wink (http://www.debugmode.com/wink/) - elaboração de tutoriais;

- Régua e Compasso (http://www.ntegravatai.relrs.g12.br/progr/ReC/) - elaboração de applets;

- GeoGebra (http://www.geogebra.org/cms/) - elaboração de applets; 
- FX - The Efeito Generator (http://www.effectgenerator.com) - desenvolvimento de pequenas animações;

- Wingeom (http://math.exeter.edu/rparris/wingeom.html) - elaboração de construções;

- Winplot (http://math.exeter.edu/rparris/winplot.html) - elaboração de construções;

- Adobe Flash (http://www.adobe.com/) - desenvolvimento do menu principal animado.

Após a identificação dos softwares, o OA foi, de fato, construído. $\mathrm{Na}$ fase de desenvolvimento foram necessárias muitas horas de trabalho e diversas reuniões para análise dos resultados parciais obtidos.

Tanto a concepção, planificação quanto a implementação do OA foram norteadas pela experiência de suas desenvolvedoras na realização de oficinas pedagógicas presenciais, utilizando softwares educacionais, destinadas a professores e licenciandos em Matemática. Conhecer o público-alvo e ter consciência dos objetivos a serem atingidos favoreceu, consideravelmente, a elaboração do SoftMat-OA.

Descrevem-se, a seguir, as seções do SoftMat-OA, assim como, os critérios de usabilidade considerados. A Figura 2 mostra a tela inicial do objeto. A imagem que aparece nessa tela não é estática. Ela surge na página e tem um efeito de mudança de cor. Todas as páginas têm cor de fundo azul claro, com detalhes em tom de azul mais escuro. A apresentação adequada das cores e suas combinações são essenciais na construção de interfaces educacionais, devendo ser consideradas as características do público-alvo, de forma que as cores possam funcionar como fonte de estímulo e motivação (Silva; Fonseca, 2002). Estas recomendações foram consideradas na escolha das cores do SoftMat-OA.



Figura 2 - Tela Inicial

Ao clicar na seta no canto inferior direito (Figura 2), o usuário é direcionado a uma página que contém um menu animado (Figura 3a). Este foi elaborado em forma de pastas (correspondentes às seções do SoftMat-OA) e é apresentado na imagem de uma tela de computador. Ao clicar em uma das pastas, esta é direcionada ao centro da tela (Figura 3b) e a seção correspondente é aberta, seqüencialmente. Buscou-se fazer um menu agradável esteticamente, a partir de um padrão que é, em geral, familiar ao usuário.

A pasta mostrada ao centro na Figura 3b, por exemplo, direciona para a página principal da seção Apresentação (Figura 4). Esta seção foi estruturada para dar boasvindas ao aluno e orientá-lo com relação à proposta da oficina, permitindo que objetivos, concepções metodológicas e critérios de avaliação estejam disponíveis. 


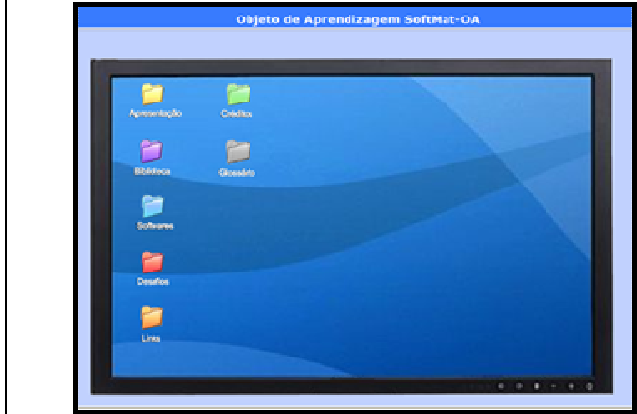

Figura $3 \mathrm{a}$

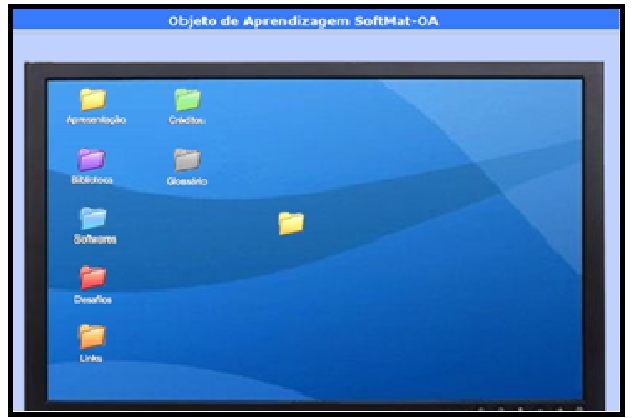

Figura $3 b$

Figura 3 - Menu Principal Animado

No topo de todas as páginas internas há sempre o logotipo do objeto (Figura 4, canto superior à esquerda), que faz link para a página inicial, e a figura do menu principal animado (Figura 4, canto superior à direita). Embora o link para o menu animado esteja sempre disponível, há sempre, ao final de cada página, um outro menu que cumpre a mesma função. Visou-se, com isso, favorecer a navegação do usuário, tornando-a mais objetiva, evitando percursos desnecessários.

As páginas são, sempre que possível, curtas. A altura das páginas é um fator que requer atenção. Páginas muito longas tornam a leitura cansativa (Sousa, 2003). Por outro lado, páginas muito curtas, que requerem vários cliques para uma leitura completa do conteúdo, podem tornar-se desestimulantes (Sousa, 2003). O ideal é alcançar um meio termo. No caso do SoftMat-OA, optou-se pela separação dos tópicos, de forma que cada página se encerrasse em si mesma, sem fazer uso de páginas longas. Pretendeu-se, assim, tornar a leitura menos cansativa.

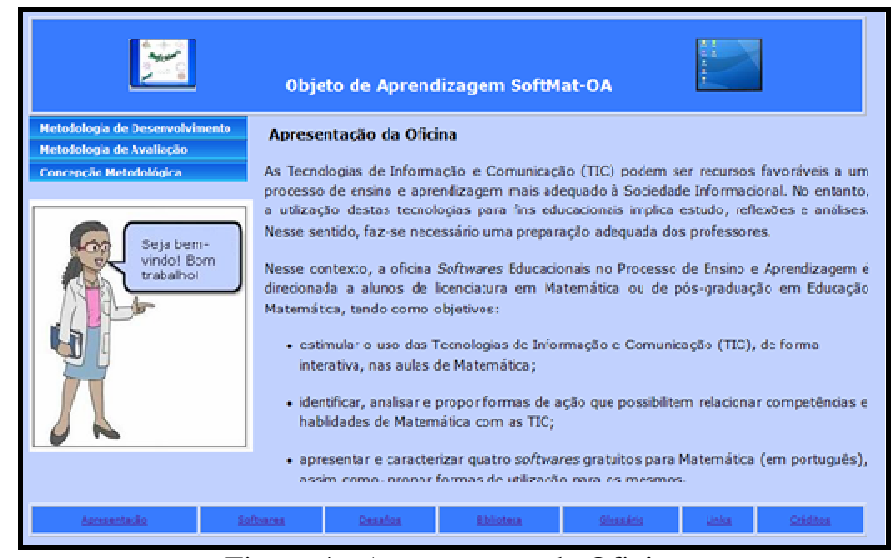

Figura 4 - Apresentação da Oficina

A principal seção do SoftMat-OA é Softwares (Figura 5). Esta foi estruturada com vistas a organizar o conteúdo pedagógico a ser abordado na oficina. A mesma foi dividida em quatro subseções, uma para cada software educacional considerado nessa proposta inicial. Cada subseção contém informações sobre o software (descrição e instalação), formas de aplicação do mesmo como recurso pedagógico (exercício, apostila de atividades, galeria de applets ou de animações, dependendo do software) e links externos para outros materiais relacionados ao software.

Na página principal dessa seção, há um menu lateral com os quatro softwares a serem trabalhados na oficina: Régua e Compasso, GeoGebra, Winplot e Wingeom (todos gratuitos e direcionados para Matemática). Clicando em Régua e Compasso, por exemplo, o usuário é direcionado para a subseção correspondente ao mesmo (Figura 6). 
Nesta, o usuário encontra um novo menu lateral (Instalação, Tutorial, Exercícios, Apostila de Atividades, Galeria de Applets, Links ReC).

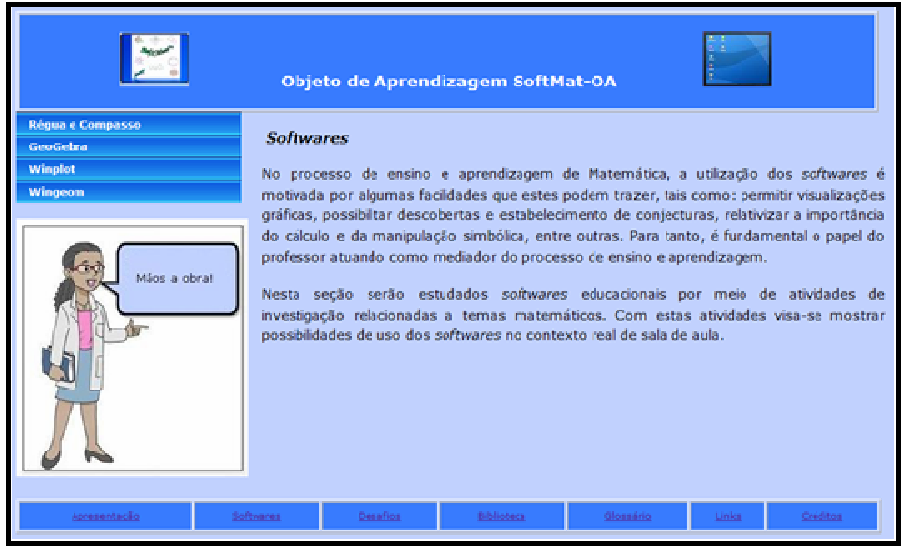

Figura 5 - Tela Inicial da Seção Software

No storyboad (Figura1) há indicação de uma subseção Descrição, mas durante a implementação do objeto, esta foi substituída pela subseção Tutorial, pois, entendeu-se que um tutorial sobre o software, com animações, seria mais favorável ao entendimento do que uma descrição textual apenas, como havia sido planejado inicialmente. No rodapé da página, há além do menu principal, um outro, permitindo acesso às subseções dos outros softwares.

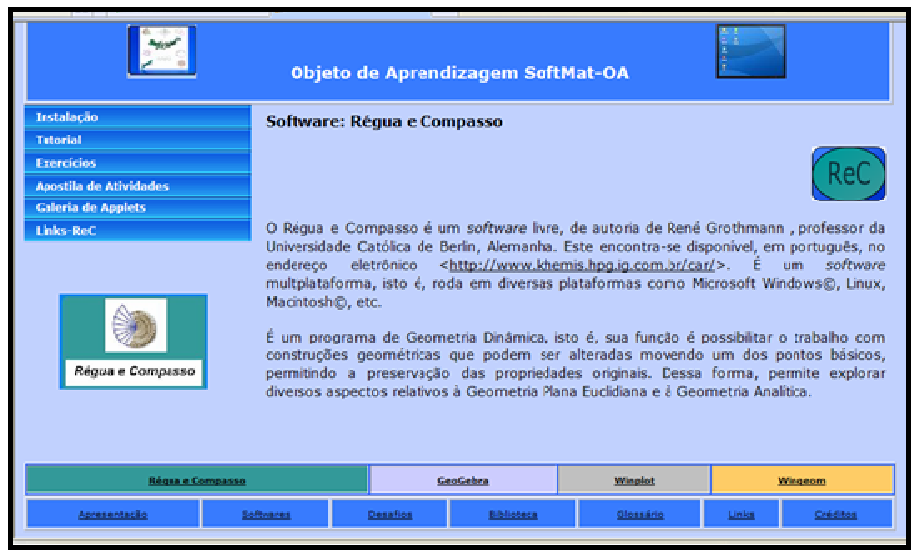

Figura 6 - Tela Inicial da subseção Software Régua e Compasso

Clicando em Exercícios, no menu correspondente a cada software, o usuário é direcionado para uma página que contém exercícios para serem resolvidos on-line (por meio de applets ou construções, previamente elaboradas). De maneira geral, objetiva-se com tais exercícios, mostrar formas práticas de utilização dos softwares. O participante da oficina não terá que desenvolver toda a construção (diferentemente das atividades da apostila) e sim, complementar construções, a partir do que for solicitado, ou estabelecer conjecturas a partir de movimentações e observações. A figura 7 mostra um exemplo de exercício que utiliza um applet elaborado no software GeoGebra.

Clicando em Apostila de Atividades, no menu correspondente a cada software, o usuário é direcionado para uma página na qual é possível obter uma apostila com uma breve descrição dos recursos do software, atividades de reconhecimento do mesmo e atividades didáticas que relacionam os recursos deste à proposta de construção do conhecimento de algum tema matemático. Nestas atividades, os participantes terão que desenvolver as construções por inteiro nos respectivos softwares, movimentá-las, 
observá-las e estabelecer conjecturas. Além da utilização na oficina em si, a apostila poderá subsidiar o uso de cada software nas práticas docentes dos participantes.

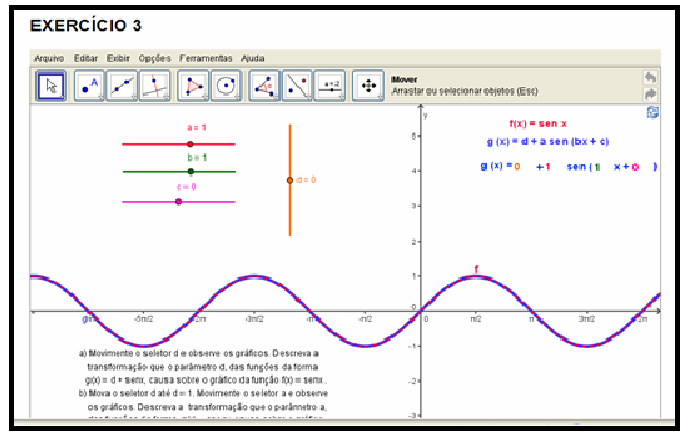

Figura 7 - Applet elaborado no GeoGebra

Ao clicar em Galeria de Applets (Régua e Compasso e GeoGebra) ou Galeria de Animações (Winplot e Wingeom), no menu correspondente a cada software, o usuário é direcionado para uma página na qual é possível acessar diversos applets ou animações relacionados a temas matemáticos diversos, possibilitando que os participantes percebam diversas formas de utilização dos softwares como recurso didático.

As demais seções do SoftMat-OA (Desafios, Biblioteca, Links, Glossário e Créditos) foram elaboradas seguindo o mesmo padrão das seções descritas. A seção Desafios foi planejada para disponibilizar tarefas, com um grau de dificuldade maior do que as atividades da seção Softwares, a serem desenvolvidas pelos alunos. São tarefas amplas visando elaboração, análise ou validação de materiais pedagógicos envolvendo o uso de algum dos quatro softwares educacionais trabalhados.

O objetivo das seções Biblioteca e Links é favorecer a leitura de materiais complementares. A Biblioteca foi idealizada para disponibilizar arquivos, em formato pdf, relacionados ao uso das TICs na Educação, de maneira geral, e, em particular, na aprendizagem de Matemática. Já a seção Links, visa a disponibilizar endereços eletrônicos relacionados aos mesmos temas da seção Biblioteca.

A seção Glossário disponibiliza definições relacionadas ao tema da oficina, buscando favorecer o entendimento do mesmo. A seção Créditos apresenta os nomes e e-mails das autoras, os softwares utilizados e agradecimentos a pessoas que, de alguma forma, colaboraram para o desenvolvimento do SoftMat-OA.

\section{Resultados e Discussão}

O SoftMat-OA foi desenvolvido no âmbito da disciplina "Tecnologias da Informação e Comunicação Aplicada à Educação" do programa de Pós Graduação em Informática na Educação/UFRGS/DINTER CEFET Campos. O referido objeto foi apresentado em um seminário e, posteriormente, avaliado por um grupo de doutorandos, alunos da disciplina em questão, com o objetivo de, em um primeiro momento, validar o objeto em sua totalidade, em nível de design, usabilidade e estrutura para aperfeiçoá-lo. Todos os avaliadores são professores do CEFET Campos, atuando em áreas distintas: Matemática, Informática, Telecomunicações e Línguas. O resultado da avaliação foi apresentado por meio de relatório, no qual foi registrado que:

- todas as seções listadas no storyboard constam no objeto desenvolvido. Sua estrutura hierárquica, no entanto, não corresponde à estrutura do objeto (que permite uma navegação não linear);

- os critérios de usabilidade, acessibilidade, navegabilidade e reusabilidade foram plenamente atendidos, enquanto requisitos no desenvolvimento de um OA; 
- o material didático pedagógico disponível no objeto tem excelente qualidade, atribuída ao tempo dedicado a este trabalho pelas elaboradoras e a pesquisas na área de Matemática realizadas pelas mesmas;

- os softwares educacionais foram bem explorados na proposta do objeto;

- os desafios propostos contribuem para incentivar o uso dos softwares e de materiais de apoio;

- o tema proposto (preparação de professores para o uso pedagógico das TICs) é muito relevante para o contexto atual.

Quanto à interface, os avaliadores sugeriram que fossem feitas algumas modificações, são elas: i) os applets deveriam ser abertos na tela do objeto e não em nova janela, de modo que os menus e barra de título continuassem aparecendo; ii) a cor das letras do menu inferior deveria ser alterada, para haver contraste com a cor de fundo, assim como, o tamanho das mesmas deveria ser aumentado; iii) uma frase do tutorial do software Wingeom, deveria ser revista.

As alterações sugeridas foram realizadas. De maneira geral, a avaliação foi bastante positiva, o que se atribui aos cuidados relativos à usabilidade da interface, à qualidade do material disponibilizado e à proposta do objeto. Futuramente, pretende-se avaliar o referido objeto, em nível de conteúdo, com professores e licenciandos em Matemática.

\section{Considerações Finais}

As TICs abrem ricas possibilidades para a educação, tais como simulação, visualização, experimentação, redução de distâncias geográficas, trabalho cooperativo, pesquisas, entre outras. No entanto, sua utilização, de forma a colaborar para a melhoria da aprendizagem, implica preparação adequada do professor. A atitude deste, enquanto mediador do processo de ensino e aprendizagem, em particular do que envolve TICs, é fundamental para obtenção de resultados positivos. O SofMat-OA pode contribuir na formação de professores de Matemática para o uso das TICs no contexto educacional. Os recursos disponibilizados no referido objeto (softwares, tutoriais, applets, atividades, entre outros) são sugestões de como o professor pode utilizar as TICs com vistas a possibilitar o estabelecimento de conjecturas, questionamentos e reflexões.

Com a descrição do objeto desenvolvido, espera-se estar divulgando idéias de como as TICs podem ser estudadas de forma a contribuir na construção de conhecimentos matemáticos. Pretende-se dar continuidade às atividades desenvolvidas, validando o SoftMat-OA em uma oficina pedagógica, presencial ou semi-presencial, tendo como público-alvo professores e licenciandos em Matemática. A oficina será realizada no âmbito do projeto de pesquisa "Tecnologias de Informação e Comunicação no Processo de Ensino e Aprendizagem de Matemática", desenvolvido no CEFET Campos e coordenado por duas das autoras deste artigo.

Finalizando, destaca-se que a concepção e desenvolvimento do SoftMat-OA exigiu muito esforço e dedicação, mas foi extremamente gratificante. A experiência foi muito rica, as tomadas de decisão exigiram reflexões e pesquisas e o desenvolvimento do objeto em si requereu o estudo de diversos softwares, assim como, de critérios de usabilidade. Os resultados da avaliação do objeto com relação ao design, usabilidade e estrutura destacam a qualidade do SoftMat-OA, decorrente de um trabalho comprometido, baseado em experiências anteriores e em ideais educacionais.

\section{Referências Bibliográficas}

BALDIN, Y. Y. Utilizações Diferenciadas de Recursos Computacionais no Ensino de Matemática. In: CARVALHO, L. M.; GUIMARÃES, L.C. (Org.). História e Tecnologia no Ensino da Matemática. Rio de Janeiro: IME-UERJ, 2002. p. 29-37. 
BARCELOS, G. T. Inovação no Sistema de Ensino: o Uso Pedagógico das Tecnologias de Informação e Comunicação nas Licenciaturas em Matemática da Região Sudeste. Dissertação (Mestrado em Ciências de Engenharia). Campos dos Goytacazes, RJ, Universidade Estadual do Norte Fluminense - UENF, 2004.

BETTIO, R. W. de; MARTINS, A. Objetos de aprendizado: um novo modelo direcionado ao ensino a distância. Documento online publicado em 17/12/ 2004: Disponível em: <http://www.universia.com.br/materia/materia.jsp?id=5938>. Acesso em: $12 / 10 / 2008$.

D’AMBRÓSIO, U. Educação Matemática: da Teoria à Prática. 8. ed. Campinas, SP: Papirus, 2001.

INEP. Tabela Resultados Internacionais. 2007. Disponível em: http://www.inep.gov.br/internacional/pisa/Novo/. Acesso em: 05/10/08.

FAGUNDES L. Podemos Vencer a Exclusão Digital. Revista Nova Escola, n.171, p. 24-26, 2004.

MACÊDO, L. N. de; CASTRO FILHO, J. A. de; MACÊDO, A. A. M.; SIQUEIRA, D. M. B.; OLIVEIRA, E. M. de; SALES, G. L.; FREIRE, R. S. Desenvolvendo O Pensamento Proporcional com o Uso de um Objeto de Aprendizagem. In: PRATA, C. L.; NASCIMENTO, A. C. A de (Orgs.) Objetos de aprendizagem: uma proposta de recurso pedagógico. Brasília: MEC, SEED, 2007. p.17-26.

MORAN, J. M. Ensino e Aprendizagem Inovadores com Tecnologias Audiovisuais e Telemáticas. In: MORAN, J.M.; MASETTO, M. T.; BEHRENS, M.A. Novas Tecnologias e Mediação Pedagógica. 5. ed. São Paulo: Papirus, 2002.

PONTE, J. P.; OLIVEIRA, H.; VARANDAS, J. M. O Contributo das Tecnologias de Informação e Comunicação para o Desenvolvimento do Conhecimento e da Identidade Profissional. In: FIORENTINI, D. (Ed.). Formação de professores de Matemática: Explorando novos caminhos com outros olhares. Campinas: Mercado de Letras, 2003. p. 159-192.

RIVED. Conheça o RIVED. s.d. Disponível em: $<$ http://www.rived.mec.gov.br/site_objeto_lis.php>. Acesso em: 02/10/08.

SETTE, S. S., AGUiAR, M. A., SETTE, J. S. A. Formação de Professores em Informática na Educação - Um Caminho para Mudanças. Coleção Informática para a Mudança na Educação. MEC/SED/PROINFO, 1999.

SILVA, A. A.; FONSECA, F. B. O poder educativo do design no e-learning. 2002. $<$ http://webinsider.uol.com.br/index.php/2002/06/17/o-poder-educativo-do-design-no-elearning/>. Acesso em 07/10/2008.

SOUSA, A. de A de. Usabilidade e a Padronização no E-learning. In: Marco Silva. (Org.). Educação Online. São Paulo: Loyola, 2003. p. 369-376.

TAROUCO, L. M. R; DUTRA, R. Padrões e Interoperabilidade. In: PRATA, C. L.; NASCIMENTO, A. C. A de (Orgs.) Objetos de aprendizagem: uma proposta de recurso pedagógico. Brasília: MEC, SEED, 2007. p. 81-92.

VYGOTSKY, L. S. A formação social da mente: o desenvolvimento dos processos psicológicos superiores. 7.ed. São Paulo, Martins Fontes, 2007.

WILEY, D. Connecting learning objects to instrucional design theory: a definition, a metaphor, and taxonomy. 2001. Disponível em: <www.reusability.org/read/chapters/wiley.doc>. Acesso em: 13/10/08. 